\title{
Microtwinning in microcrystalline silicon and its effect on grain-size measurements
}

\author{
L. Houben, ${ }^{1}$ M. Luysberg, ${ }^{1}$ and R. Carius ${ }^{2}$ \\ ${ }^{1}$ Institut für Festkörperforschung, Forschungszentrum Jülich, D-52425 Jülich, Germany \\ ${ }^{2}$ Institut für Photovoltaik, Forschungszentrum Jülich, D-52425 Jülich, Germany \\ (Received 9 May 2001; revised manuscript received 25 October 2002; published 16 January 2003)
}

\begin{abstract}
Microcrystalline silicon grown by plasma-enhanced chemical vapor deposition consists of a phase mixture of amorphous silicon and submicrometer sized columnar crystalline silicon grains. These grains do not exhibit perfect translational symmetry but show a high density of microtwins, evidenced by structural investigations by use of transmission electron microscopy and x-ray diffraction. The high twin fault density of typically every tenth $\{111\}$ bilayer leads to anisotropic size broadening effects for Bragg reflection peaks. This anisotropic behavior of size broadening interferes with grain-size and strain measurements from x-ray diffractograms. Experimentally measured apparent grain sizes are up to a few ten nanometers and are subject to significant scatter due to the effect of microtwinning. Numerical modeling of the shape of Bragg reflection peaks is presented for the two characteristic modifications of microtwinning, i.e., lamellar and random twinning, to assess quantitatively the effect of microtwinning on size broadening and grain-size measurements from Bragg reflection width.
\end{abstract}

DOI: 10.1103/PhysRevB.67.045312

PACS number(s): 68.55.Jk, 61.10.Nz, 68.37.Lp

\section{INTRODUCTION}

Microcrystalline silicon ( $\mu c-\mathrm{Si}: \mathrm{H})$ is a prospective material for the application in large scale microelectronics prepared in a low-temperature process $\left(\leqslant 400^{\circ} \mathrm{C}\right)$ on inexpensive substrates. Presently, extensive work covers the development of stacked thin film solar cells employing a $\mu c-\mathrm{Si}: \mathrm{H}$ absorber layer. ${ }^{1-3}$

The preferred technique for the deposition of $\mu c-\mathrm{Si}: \mathrm{H}$ is plasma-enhanced chemical vapor deposition (PECVD), using silane highly diluted in hydrogen as source gases. Depending on the detailed deposition parameters, microcrystalline silicon growth leads to a variety of compositional and microstructural properties. ${ }^{4-6}$ Generally, $\mu c$-Si:H consists of a heterogeneous phase mixture of amorphous silicon and submicrometer sized crystalline grains in variable volume fractions and a considerable fraction of disordered material located at grain boundaries.

The specific structural properties of $\mu c-\mathrm{Si}: \mathrm{H}$ are of interest regarding a proper interpretation of structure sensitive experimental data and an understanding of the growth kinetics. A careful interpretation of, e.g., Raman spectra used as a standard characterization tool relies on a sensible distinction of disorder induced effects due to lattice strain and the smallness of grain sizes and their distribution. ${ }^{7-9}$ Furthermore, several growth models proposed in the literature ${ }^{4,10-12}$ remain hypothetical up to now, principally due to missing provision for the formation kinetics reflected in the heterogeneous microstructure of the microcrystalline films. Even so, to our knowledge, there has been no thorough compilation of microscopic features of $\mu c-\mathrm{Si}: \mathrm{H}$ that could even serve for a consistent picture of grain boundaries and grain sizes in $\mu c-\mathrm{Si}: \mathrm{H}$.

In this paper, we focus on recurring characteristics of the crystalline phase and the grain boundaries that are, to a large extent, independent of the growth conditions. A classification of the characteristic crystallographic defects within the crystalline grains is derived from structural investigations by transmission electron microscopy (TEM) in the first part of the paper. The primary form of disorder within the crystalline grains is polytypism due to microtwinning. Its broadening effect on the Bragg reflection peaks in x-ray diffraction (XRD) and thus on grain-size quantification is investigated in a second part of the paper. Numerical calculations for two characteristic disorder patterns, one-dimensional twinning on $\{111\}$ lattice planes, and three-dimensional twinning on random $\{111\}$ lattice planes, are presented to provide a better understanding of what can be deduced about grain sizes and strain estimates for $\mu c-\mathrm{Si}: \mathrm{H}$ and related materials from $\mathrm{x}$-ray diffraction patterns.

\section{EXPERIMENT}

The microcrystalline silicon samples were prepared in a conventional diode-type reactor using very high frequency PECVD at $95 \mathrm{MHz}$ at a plasma power up to $50 \mathrm{~W}$. Only samples with high crystalline volume fraction will be considered here. The detailed preparation parameters are similar to those given in Refs. 13 and 14 for highly crystalline samples. In general, the $\mathrm{SiH}_{4}$ source gas was highly diluted in $\mathrm{H}_{2}$. Doped and undoped samples were deposited onto Corning 7059 glass substrates and onto silicon substrates with native oxide layer at a substrate temperature of less than $230{ }^{\circ} \mathrm{C}$. The film thicknesses were typically in the order of a few 100 $\mathrm{nm}$ to about $3 \mu \mathrm{m}$.

For TEM investigation, cross-sectional specimen were prepared by standard mechanical procedures followed by Ar ion milling. Conventional Bragg contrast images were recorded with a JEOL 2000EX microscope at an accelerating voltage of $200 \mathrm{kV}$, high-resolution lattice fringe images were taken using a JEOL 4000EX microscope operated at $400 \mathrm{kV}$.

XRD analyses were carried out with a conventional $\mathrm{Cu}-K_{\alpha}$ x-ray radiation source using a symmetric BraggBrentano powder diffraction setup for $\Theta-2 \Theta$ scans and a parallel beam diffraction setup under grazing incidence ${ }^{15,16}$ in reflection. Grazing incidence XRD was applied to enhance 


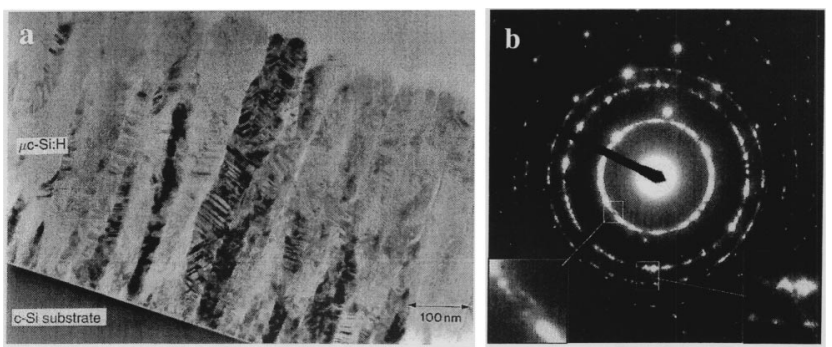

FIG. 1. (a) Cross-sectional TEM bright-field micrograph showing a microcrystalline silicon film with low amorphous volume fraction. The irregular fringe contrast originates from fine twin lamellae with a typical size of a few nm perpendicular to the fault plane. (b) Selected area diffraction pattern corresponding to the image in (a), taken in the substrate's [110] orientation. The innermost Debye-Scherrer ring is a double ring made up of $\{111\}$ lattice reflections of diamond cubic silicon and extra reflections from twin planes (enlarged in the inset left). Fine streaks run along $\langle 111\rangle$ directions through the reflections (enlarged in the inset right).

the surface sensitivity and thereby allowing for the investigation of films as thin as a few hundred nm. Here, an incident angle larger than the critical angle $\alpha_{C}=0.22^{\circ}$ for total reflection but well below one degree was applied, assuring that the penetration depth for the $\mathrm{x}$ rays was about equal to the film thickness. The instrumental broadening was measured from a NIST SRM 640a silicon powder sample. The standard's profile shapes were used in a Stokes correction (see Warren ${ }^{24}$ for details) for the evaluation of the shape of the lattice reflections.

\section{RESULTS}

\section{A. Grain boundaries and domain structure in $\mu c-\mathrm{Si}: \mathrm{H}$ crystalline grains}

Figure 1(a) shows a bright-field image of a $\mu c-\mathrm{Si}: \mathrm{H}$ film with low amorphous volume fraction. The film was grown at a high input power of $50 \mathrm{~W}$ and a silane concentration of $2 \%$ on the native oxide of a $\mathrm{Si}(100)$ wafer. The characteristic columnar structure of highly crystalline $\mu c$-Si:H (Refs. 4-6) is well developed under these conditions. The crystalline columns consist of coherent domains defined by fine twin lamellae, causing the characteristic irregular fringe contrast. Twin planes are repeated not strictly periodically in short distance along the fault normal. Typically, the size of a single twin domain is as small as a few $\mathrm{nm}$ perpendicular to the twin plane and up to a few ten nm on that plane. Measuring the twin fault probability $\lambda$ in two of the columns in Fig. 1(a) gives $\lambda \approx 0.1$, i.e., there is a twin boundary every ten (111) bilayers. It can be concluded from our TEM investigations that this value for $\lambda$ is typical for microcrystalline silicon, even when smaller columnar structures or amorphous volumes are present.

The selected area diffraction pattern (SADP) corresponding to the image in Fig. 1(a) is given in Fig. 1(b). More than one column contributes to the SADP due to a selected area of $\approx 500 \mathrm{~nm}$ in diameter. The random placement of diffraction spots on a certain Debye-Scherrer ring implies that there is no orientational relationship between neighboring crystalline columns. Thus, the grain boundaries separating adjacent columns have to be considered as incoherent. The fine structure in this reciprocal space map reveals fine lines passing through many diffraction spots. An example can be seen in the enlarged part on the right-hand side of Fig. 1(b). The streaks are caused by the planar interfaces of the twin lamellae and thus visualize the broadening effect on Bragg reflection peaks due to the small coherently scattering twin domains. The streaks run along $\langle 111\rangle$ directions relative to the diffraction vector, which lies approximately in the plane of the SADP as does the streak direction. The corresponding grain can be identified with a column showing a strong fringe contrast from twin lamellae. Some of the streaks are found to be composed of closely spaced spots, indicating coherent interference of electron waves from closely spaced microtwins along a common $\langle 111\rangle$ fault normal, but not necessarily a periodic superstructure. ${ }^{17}$

The diffraction spots are displaced relative to their position in a perfect lattice since the Bragg peaks appear where a reciprocal lattice streak passes through the dispersion sphere of the electrons. This is illustrated in more detail in Appendix A, which gives also an estimate for the expectation value for the scatter $\varepsilon$ of the reciprocal lattice plane distance of a certain reflection $\vec{g}_{h k l}$ relative to that in the perfect lattice of bulk silicon. $\varepsilon$ can be roughly estimated by a linear approximation to $\varepsilon \approx \lambda\left[(h+k+l) /\left(h^{2}+k^{2}+l^{2}\right)\right]$ for twinning on the (111) lattice planes. For example, $\varepsilon$ is about $5 \%$ for $\lambda$ $=0.1$ and $h k l=220$. A displacement of diffraction spots of this magnitude can thus be due to anisotropic size broadening only and is not necessarily indicative of lattice strain.

The innermost ring of reflections is a double ring. This becomes evident from the left-hand side inset in Fig. 1(b). The outer ring can be attributed to the $\{111\}$ lattice spacing in diamond cubic silicon $(d c-\mathrm{Si})$. The inner ring has a reciprocal lattice distance $q \approx 3.05 \mathrm{~nm}^{-1}$ close to a scattering vector $\frac{1}{3} q_{422}=3.007 \mathrm{~nm}^{-1}$. The latter reflection is a forbidden reflection for $d c-\mathrm{Si}$ in the kinematic approximation, but it appears because of the hexagonal stacking sequence around a stacking fault. ${ }^{18}$ Attributing the extra reflection to hexagonal $\mathrm{Si}$ is consistent with the interpretation of Hendriks et al., ${ }^{19}$ who analyzed films grown by low pressure chemical vapor deposition (LPCVD) in the temperature range between 560 and $1000{ }^{\circ} \mathrm{C}$. Hendriks et al. ${ }^{19}$ relate extra reflections in their $\mathrm{x}$-ray diffractograms to polytypic series of diamond hexagonal silicon $(d h-\mathrm{Si})$. For these polytypic modifications, the $\{01 \overline{1} 0\}$ reflection in pure diamond hexagonal silicon $(d h-\mathrm{Si})$ has the largest lattice spacing of $0.323 \mathrm{~nm}^{-1}$, i.e., the smallest scattering vector $q=3.007 \mathrm{~nm}$. Depending on their periodicity, the polytypic modifications show a number of lattice spacings inbetween the spacing of the $\{01 \overline{1} 0\}$ lattice planes of $d h-\mathrm{Si}$ and the $\{111\}$ lattice planes of $d c-\mathrm{Si}$, leading to a shift and broadening of the extra peak towards the $\{111\}$ reflection of $d c$-Si in the x-ray diffraction patterns and additional spots on electron diffraction patterns with a multitude of lattice spacings larger than the $\{111\}$ lattice spacing in $d c-\mathrm{Si}^{19,20}$

Further support for the polytypic modifications is given by Raman spectroscopic data. Raman spectra of $\mu c-\mathrm{Si}: \mathrm{H}$ 


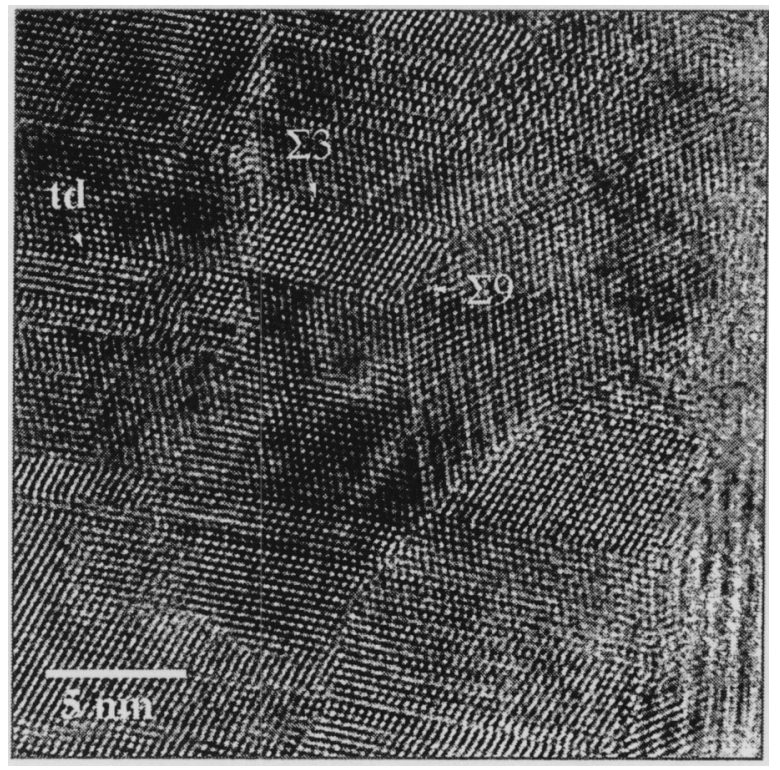

FIG. 2. High-resolution micrograph showing microtwins with a common [110] axis within a single crystalline columnar grain in $\mu c-\mathrm{Si}: \mathrm{H}$. See text for details.

show generally a Raman active vibrational mode at $\approx 495 \mathrm{~cm}^{-1}$, which is characteristic for hexagonal silicon and absent in cubic silicon. ${ }^{21}$

The polytypes can be imaged directly on high-resolution TEM micrographs. The high-resolution image in Figure 2 shows the inner part of a crystalline column as shown in Fig. 1(a). The crystalline domains can be readily identified by the traces of their $\{111\}$ lattice planes. The image shows numerous twin domains only a few $\mathrm{nm}$ in size in a projection close to a common [110] axis. Mainly coherent $\Sigma=3$ boundaries (the grain boundaries are classified according to the coincidence site lattice notation) are formed by twinning, but multiple twinning also leads to higher order twins, i.e., $\Sigma=9$ and $\Sigma=27$ boundaries. The twin domains and the twin boundaries are usually free of dislocations, the twin dislocation (TD) marked in Fig. 2 being an exception to that rule. Neither in this image nor in other high-resolution images of various $\mu c$-Si:H samples we were able to find small inclusions or bands of $d h-\mathrm{Si}$. The presence of $d h-\mathrm{Si}$ in $\mu c-\mathrm{Si}: \mathrm{H}$ is thus restricted to the local hexagonal stacking sequence around twin boundaries. The formation of these twin boundaries is favored in comparison to the formation of stacking faults, which are less prominent.

Furthermore, we did not observe any different type of grain boundary within the crystalline columns apart from the simple symmetrical twin boundary and the boundaries that are formed by intersecting twin domains. Extended hydrogen platelets, as found in the single crystalline substrate after film growth by PECVD under identical deposition conditions, ${ }^{22}$ were not detected within the crystalline grains.

In brief summary of the results of the TEM investigations, our highly crystalline PECVD grown $\mu c$-Si:H consists of columnar shaped single crystal grains, separated by incoherent grain boundaries. The inner structure of the grains is characterized by multiple twinning. Mainly coherent $\Sigma 3$
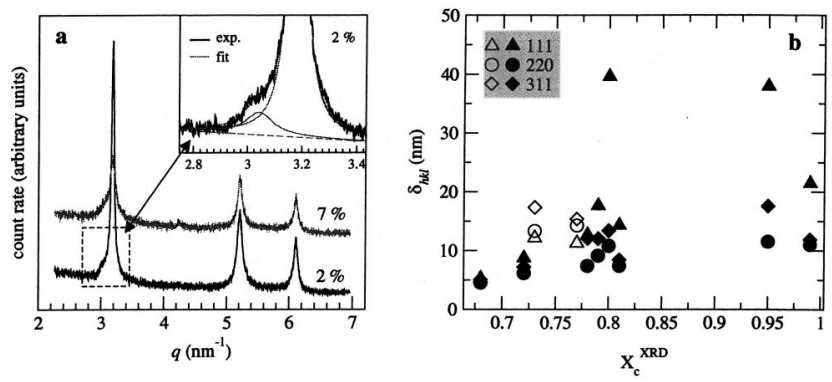

FIG. 3. (a) X-ray diffractograms of two $\mu c-\mathrm{Si}: \mathrm{H}$ samples, deposited at the indicated silane concentration in the gas phase. The diffractograms were recorded in a grazing incidence setup. (b) Apparent grain sizes $\delta_{h k l}$ evaluated from reflection widths for a series of microcrystalline samples with different crystalline volume fraction. Two characteristic orderings can be distinguished: $\delta_{111} \gg \delta_{311}$ $>\delta_{220}$ (filled symbols) and $\delta_{311}>\delta_{220}>\delta_{111}$ (open symbols).

twin boundaries on $\{111\}$ lattice planes are found and a preference for lamellar twin patterns can be seen. A twin faulting with probability $\lambda$ as high as 0.1 leads to a considerable broadening of the reciprocal space features like Bragg reflections along $\langle 111\rangle$ directions, that becomes visible in selected area diffraction patterns.

\section{B. Grain size and domain size measurement}

$\mathrm{X}$-ray diffraction is routinely used to determine grain sizes $\delta$ as well as preferential growth directions in polycrystalline materials. Additionally, strain estimates can be made when comparing the width of lattice reflections and their shift, respectively. However, the perturbance of the reciprocal lattice points of domain structured nanocrystals has important consequences: Peak shapes, intensities and widths are likely to differ from extrapolated bulk estimates. In the following, this will be demonstrated for our microcrystalline samples by a numerical simulation based on a phenomenological approach, which is compared to experimental data. In particular, the implication of twin faulting for the peak width of Bragg reflections and therefore for the so-called apparent $^{23}$ grain size $\delta$ will be described in detail.

\section{Experimental $x$-ray diffraction data}

In Fig. 3(a) X-ray diffractograms of the $\mu c-\mathrm{Si}: \mathrm{H}$ samples are shown, measured in grazing incidence at an angle of $\approx 0.3^{\circ}$. The samples covered by Fig. 3(a) were grown at high and low silane concentration in the gas phase, respectively. They can be considered as representative for two regimes of microcrystalline silicon growth yielding distinctively different column sizes of crystalline grains. From TEM imaging, it is known that both films show a columnar structure with high crystalline volume fraction, but there is a decrease in the typical column diameter from $\approx 50 \mathrm{~nm}$ at a silane concentration of $2 \%$ to $\approx 20 \mathrm{~nm}$ at a silane concentration of $7 \%$. The decrease of the column size is characteristic of the growth of $\mu c-\mathrm{Si}: \mathrm{H}$ when approaching the transition to amorphous silicon growth by increasing the silane concentration in the gas phase. ${ }^{6}$ 
Both films show distinct $\{111\},\{220\}$, and $\{311\}$ lattice reflections, indicating the high degree of crystallinity. Apparently, there is an additional reflection of $d h$-Si at $q$ $\approx 3.04 \mathrm{~nm}^{-1}$ related to the twin boundaries for the sample deposited at lower silane concentration. The inset in Fig. 3(a) shows the magnified $\{111\}$ Bragg reflection peak in the sample deposited at a silane concentration of $2 \%$ together with Lorentzian fit profiles for the $\{111\}$ reflection of $d c-\mathrm{Si}$ and the extra reflection on top of a parabolic background. For the higher silane concentration, the additional reflection is obscured in the tail of the broader $\{111\}$ reflection of the $d c$-Si. The intensity of the additional reflection could be used to estimate the density of twin boundaries. However, a quantitative consideration of the intensity of the additional reflection will not be given since the data shown here do not provide a stable numerical fit. The stable numerical fit would be required since the intensity and shape of the additional reflection are not known from theoretical consideration. The shape depends on the occurrence of the various polytype modifications, each of which gives rise to diffraction intensity at a certain lattice spacing smaller than the $\{111\}$ spacing in $d c-\mathrm{Si}^{19,20} \mathrm{~A}$ more stable quantification of the average twin density can be expected from the evaluation of grain sizes, more precisely the shape the shape transform in the diffraction intensity.

Careful inspection of the lattice reflections of the $d c-\mathrm{Si}$ reveals a specific feature common to highly crystalline $\mu c$-Si:H films: The integral width $\beta_{h k l}$, i.e., the area divided by the amplitude, of the three most prominent lattice reflections increases in the order $\beta_{111} \ll \beta_{311}<\beta_{220}$. For example, the film deposited at $2 \%$ silane concentration in Fig. 3(a) $\beta_{111}: \beta_{311}: \beta_{220}=0.024 \mathrm{~nm}^{-1}: 0.058 \mathrm{~nm}^{-1}: 0.083 \mathrm{~nm}^{-1}$ after a Stokes correction ${ }^{24}$ for the instrumental broadening. The apparent grain size $\delta_{h k l}=\beta^{-1}{ }_{h k l}$ evaluated from the width of the lattice reflections is thus strongly anisotropic. $\beta_{111}$ is considerably smaller than both $\beta_{220}$ and $\beta_{311}$ and there is no monotoneous order with increasing scattering angle, contradicting the presence of a homogeneous microstrain, for which the broadening would be simply proportional to the magnitude of the scattering vector $\vec{q}$ (see Langford, ${ }^{23}$ and references therein). In addition, the ratio of the integrated intensities $I_{111}: I_{220}: I_{311}$ is close to that of an ideal powder sample consisting of single crystalline grains, contradicting an explanation by preferential growth directions. A slight deviation from the ideal intensity ratios is simply explained when accounting for the intensity of the extra twin reflection which diminishes the intensity of the $\{111\}$ reflection of $d c$ Si.

Further data for apparent grain sizes in $\mu c-\mathrm{Si}: \mathrm{H}$ is shown in the scatter graph in Fig. 3(b) for several highly crystalline microcrystalline samples. Here, the apparent grain size $\delta_{h k l}$ $=\beta^{-1}{ }_{h k l}$ is plotted against a measure for the crystalline volume fraction $X_{\mathrm{C}}^{X R D}$ evaluated from the diffractograms according to a method described in Ref. 6. At first glance, the data shows large scatter. In general, the $\delta_{h k l}$ increase with increasing crystallinity and the discrepancy between the $\delta_{h k l}$ for the different lattice planes $h k l$ becomes larger the higher $X_{\mathrm{C}}^{X R D}$, measuring up to a factor of 3.6. The scatter in the data can be classified in the following way: (1) the apparent grain size $\delta_{h k l}$ decreases in the order $\delta_{111} \gg \delta_{311}>\delta_{220}$ [filled symbols in Fig. 3(b)] and (2) $\delta_{h k l}$ decreases in the order $\delta_{311}$ $>\delta_{220}>\delta_{111}$ [open symbols in Fig. 3(b)].

\section{Numerical simulation for the anisotropic size broadening}

One mechanism that can lead to this kind of anisotropic broadening is microtwinning. The effect of microtwinning will now be investigated with a numerical model for the shape of the Bragg reflections. Details of the procedure are lined out in Appendix B. Before presenting the results of our computations, the terminology and basic considerations are addressed.

Starting point is the double sum which describes the coherent diffraction intensity $I$ from a crystal with atoms at positions $\vec{R}_{n}, n=\left(n_{1}, n_{2}, n_{3}\right)$, in kinematic approximation at large distance from elastically scattering centers:

$I=I_{0} \sum_{n} f_{n} \exp \left(2 \pi i \vec{q} \cdot \vec{R}_{n}\right) \sum_{n^{\prime}} f_{n^{\prime}} * \exp \left(-2 \pi i \vec{q} \cdot \vec{R}_{n^{\prime}}\right)$.

Here, $\vec{q}$ is the scattering vector and $f_{n}$ the atomic scattering factor of the atom at $\vec{R}_{n}$. Expressing the scattering vector as the sum of a reciprocal lattice vector $\vec{g}$ and the excitation error $\vec{s}, \vec{q}=\vec{g}+\vec{s}$, one obtains for a perfect parallelopipedon crystal measuring $N_{1} \times N_{2} \times N_{3}$ unit cells with orthogonal unit cell vectors with magnitude $\left(a_{1}, a_{2}, a_{3}\right)$ the Laue interference function

$$
I[\vec{s}(\vec{q}, \vec{g})] \sim|F(\vec{g})|^{2}|G(\vec{s})|^{2}=|F(\vec{g})|^{2} \sum_{i=1}^{3} \frac{\sin ^{2}\left(\pi s_{i} N_{i} a_{i}\right)}{\left(\pi s_{i} a_{i}\right)^{2}}
$$

with $F$ being the structure factor of a unit cell and $G$ the so-called shape transform. Given the crystal dimensions $N_{i} a_{i}, i=1,2,3$, and the orientation of the crystal with respect to the incident radiation one can easily calculate the shape transform and thus the Fraunhofer diffraction pattern $I[\vec{s}(\vec{q}, \vec{g})]$.

According to the previous section $\mu c-\mathrm{Si}: \mathrm{H}$ consists of a large number of small grains with nonuniform orientation, each of which shows a subdomain structure because of twin boundaries on $\{111\}$ lattice planes. This implies, that firstly the shape factor will vary from grain to grain due to the diversity of twin patterns and secondly the measured signal represents the average shape factor $\left\langle|G(\vec{s})|^{2}\right\rangle_{p}$ for an appropriate probability distribution $p(\vec{s})$ of excitation errors $\vec{s}$.

The general procedure used here to approximate $\left\langle|G(\vec{s})|^{2}\right\rangle_{p}$ is described in Appendix B. Only the phenomenological approach providing for a numerically computable representation of $\left\langle|G(\vec{s})|^{2}\right\rangle_{p}$ is given here. (1) The individual grain size is replaced by its average value, labeled $\delta_{\text {iso }}$, where the subscript "iso" indicates isotropic broadening. (2) The individual pattern of twin boundaries within a grain is simplified to a mean frequency $\delta_{\text {aniso, }}^{-1}$ synonymous to a mean reciprocal size of twin domains. (3) The broadening of the reciprocal lattice points is assumed to average to a Gauss- 


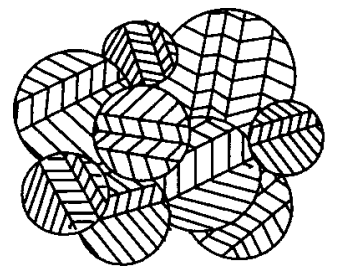

(a)

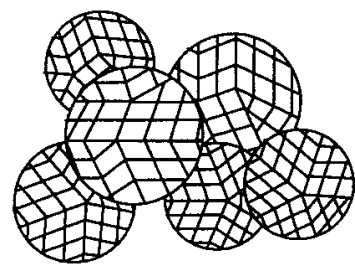

(b)
FIG. 4. The two different models used for the numerical simulation of XRD patterns of $\mu c-\mathrm{Si}: \mathrm{H}$. (a) Spherical grains with a lamellar twin (LT) structure and (b) spherical grains with random twinning (RT).

ian distribution with a full width at half maximum (FWHM) of $\delta_{\text {iso }}^{-1}$ and $\delta_{\text {aniso }}^{-1}$ for isotropic and anisotropic size broadening, respectively. (4) Diffraction from different grains is considered to be incoherent, since there is no correlation between the orientation of neighboring grains and the resolution of the diffraction pattern is limited. Thus the intensity of the x-ray diffraction patterns of two grains is the sum of the patterns of the individual grains.

The orientation of an individual crystallite is assumed to be random for two special cases displayed in Fig. 4. First, we consider a set of randomly oriented spherical grains with lamellar twinning [Fig. 4(a)] on a (111) plane ("lamellar twinned particles"). Secondly, we describe a set of randomly oriented spherical grain with random twinning [Fig. 4(b)] on any of the $\{111\}$ planes ("randomly twinned particles"). The two models are considered to represent the two extreme cases for microtwinning in a three-dimensional microcrystalline silicon grain.

Figures 5(a) and 5(c) show exemplary results for the averaged square of the shape factor $\left\langle|G(\vec{s})|^{2}\right\rangle_{p}$ for the lamellar twinned particles and the randomly twinned particles for the three lowest indexed Bragg reflections in cubic silicon. The $\left\langle|G(\vec{s})|^{2}\right\rangle_{p}$ for a fixed mean grain size $\delta_{\text {iso }}=20 \mathrm{~nm}$ and various mean twin domain sizes $\delta_{\text {aniso }}$ are normalized with respect to their area in this representation. Therefore, the amplitude of a peak directly equals the apparent grain size $\delta_{h k l}$. The arrows indicate the decrease of $\delta_{\text {aniso }}$ from infinite to 10 $\mathrm{nm}$ to $5 \mathrm{~nm}$ to $3 \mathrm{~nm}$.

The center position of the reflections is shifted insignificantly compared to the broadening even for the smallest domain size $\delta_{\text {aniso }}$, giving further evidence that the extra reflection observed at $q \approx 3.04 \mathrm{~nm}^{-1}$ is not due to a particular class of small grains but originates from $d h-\mathrm{Si}$ with a structure factor different from $d c-\mathrm{Si}$.

The change in profile width with decreasing $\delta_{\text {aniso }}$ is most prominent for the $\{220\}$ reflection. Comparing Figs. 5(a) and $5(\mathrm{c})$ one can see that the profile shape is dominated by a pronounced widening of the tails for the $\{220\}$ and the $\{311\}$ reflection for the lamellar twinned particles [Fig. 5(a)]. In contrast to the randomly twinned particles there are lattice planes in the lamellar twinned particles, which are not affected by twinning (see Table I in Appendix B) providing for a nearly constant shape around the profile center of the $\{220\}$ and the $\{311\}$ reflection at the smallest $\delta_{\text {aniso }}$.
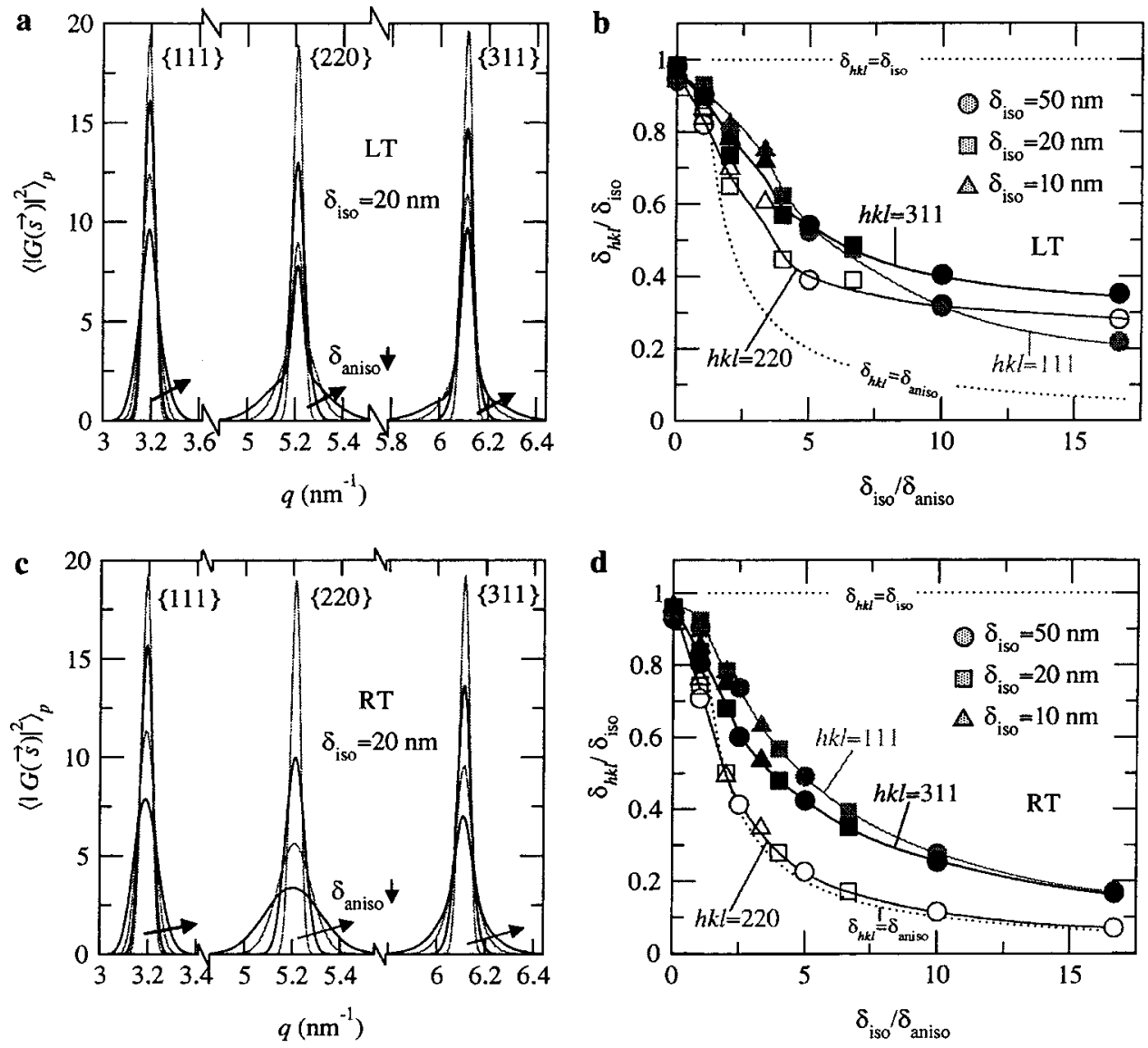

FIG. 5. The averaged square of the shape factor $\left\langle|G(\vec{s})|^{2}\right\rangle_{p}$ for a diamond cubic lattice with anisotropic broadening along $\langle 111\rangle$ directions for (a) randomly oriented lamellar twinned particles (LT) and (c) randomly oriented particles with random twinning (RT). (b) and (d) shows the apparent grain size normalized to the "true" grain size, $\delta_{h k l} / \delta_{\text {iso }}$, plotted against a measure for the number of twins within a grain $\delta_{\text {iso }} / \delta_{\text {aniso }}$ for various "true" grain sizes $\delta_{\text {iso }}$. (b) shows the case of randomly oriented lamellar twinned particles and (d) for randomly oriented particles with random twinning. The splines approximating the data points in figures (b) and (d) are guides to the eye only. The dotted lines mark where the apparent grain size is equal to the "true" grain size or the twin domain size, respectively. 
TABLE I. Twin planes in an fcc lattice, shift vectors $\Delta \vec{r}$ to faulted atom positions and phase shifts in fractions of $2 \pi$ caused by faulting for the three lowest indexed reciprocal lattice vectors in a diamond cubic lattice.

\begin{tabular}{clccc}
\hline \hline twin plane & \multicolumn{1}{c}{$\Delta \vec{r}$} & $\Delta \vec{r}\langle 111\rangle$ & $\Delta \vec{r}\langle 220\rangle$ & $\Delta \vec{r}\langle 311\rangle$ \\
\hline$(111)$ & $\frac{1}{6}\langle 11 \overline{2}\rangle, \frac{1}{6}\langle 1 \overline{2} 1\rangle, \frac{1}{6}\langle\overline{2} 11\rangle$ & $0,0,0$ & $\frac{2}{3},-\frac{1}{3},-\frac{1}{3}$ & $\frac{1}{3}, \frac{1}{3}, \frac{2}{3}$ \\
$(11 \overline{1})$ & $\frac{1}{6}\langle 112\rangle, \frac{1}{6}\langle\overline{1} 21\rangle, \frac{1}{6}\langle\overline{2} 1 \overline{1}\rangle$ & $\frac{2}{3}, \frac{1}{3},-\frac{1}{3}$ & $\frac{2}{3}, \frac{1}{3},-\frac{1}{3}$ & $1,0,-\frac{2}{3}$ \\
$(1 \overline{1} 1)$ & $\frac{1}{6}\langle\overline{1} 12\rangle, \frac{1}{6}\langle 121\rangle, \frac{1}{6}\langle 21 \overline{1}\rangle$ & $\frac{1}{3}, \frac{2}{3}, \frac{1}{3}$ & $0,2,2$ & $0,2,2$ \\
$(\overline{1} 11)$ & $\frac{1}{6}\langle 1 \overline{1} 2\rangle, \frac{1}{6}\langle 12 \overline{1}\rangle, \frac{1}{6}\langle 211\rangle$ & $\frac{1}{3}, \frac{1}{3}, \frac{2}{3}$ & $0,2,2$ & $\frac{2}{3}, \frac{2}{3}, \frac{4}{3}$ \\
\hline \hline
\end{tabular}

A quantitative evaluation of the apparent grain size $\delta_{h k l}$ normalized to the "true" grain size $\delta_{\text {iso }}$ is shown in Figs. $5(\mathrm{~b})$ and $5(\mathrm{~d})$ for various values of the grain size $\delta_{\text {iso }} . \delta_{h k l}$ normalized to $\delta_{\text {iso }}$ can be interpreted as a "squeeze" factor for the measured apparent grain size against the "true" grain size. This "squeeze" factor is plotted against the ratio of the "true" grain size and the twin domain size $\delta_{\text {iso }} / \delta_{\text {aniso }}$, i.e., a measure for the number of twins within a grain. The dotted lines in Figs. 5(b) and (d) mark where the measured apparent grain sizes $\delta_{h k l}$ would equal the twin domain size $\delta_{\text {aniso }}$ or the "true" grain size $\delta_{\text {iso }}$, respectively.

In general, the apparent grain sizes $\delta_{h k l}$ neither equals the "true" grain size $\delta_{\text {iso }}$ nor the twin domain size $\delta_{\text {aniso }}$. Only for the randomly oriented particles with random twinning, the inverse width of the $\{220\}$ reflection $\delta_{220}$ is close to the twin domain size $\delta_{\text {aniso }}$. For the randomly twinned particles, the relation $\delta_{111}>\delta_{311} \gg \delta_{220}$ holds over the whole range of $\delta_{\text {iso }} / \delta_{\text {aniso }}$ shown here. The maximum difference between the apparent grain sizes $\delta_{h k l}$ is up to a factor of 2.6 for this case. For the lamellar twinned particles, the broadening is less pronounced for the $\{220\}$ and the $\{311\}$ reflection when compared to the randomly twinned particles. A maximum deviation between the apparent grain sizes $\delta_{h k l}$ by a factor of 1.7 is found in the region of $\delta_{\text {iso }} / \delta_{\text {aniso }}$ shown here. For small grain sizes $\delta_{\text {iso }}$ or large $\delta_{\text {aniso }}$, i.e., low twin density, one finds that $\delta_{111}>\delta_{311} \gg \delta_{220}$ similar to the randomly twinned particles. Contrary to the randomly twinned particles the order of the $\delta_{h k l}$ is altered to $\delta_{311}>\delta_{220}>\delta_{111}$ for larger grain sizes and higher twin density. Thus, the two characteristic orderings in the apparent grain sizes seen in the experimental data are reproduced in the numerical data for the shape factor $\left\langle|G(\vec{s})|^{2}\right\rangle_{p}$. Furthermore, the numerical data show the increase of the scatter of the $\delta_{h k l}$ with increasing grain size $\delta_{\text {iso }}$ for a certain twin domain size $\delta_{\text {aniso }}$. In contrast, the discrepancy between the $\delta_{h k l}$ and the broadening of the $\{311\}$ reflection is more pronounced in the experimental data especially for larger $X_{C}^{\mathrm{XRD}}$. The difference is possibly due to strain broadening which becomes more prominent at larger particle size.

\section{DISCUSSION}

Our microscopical investigation of crystalline grains in $\mu c-\mathrm{Si}: \mathrm{H}$ demonstrates, that a grain in $\mu c-\mathrm{Si}: \mathrm{H}$ can be considered as a single crystal of columnar shape with faulting on $\{111\}$ lattice planes. A high stacking fault probability is well known for $\mathrm{SiC}$, where the stacking fault energy is about $2.9 \mathrm{~mJ} \mathrm{~m}^{-2}$ for the $6 \mathrm{H}$ polytype, ${ }^{25}$ but is surprisingly high for the thin silicon films grown at low temperature due to a considerable intrinsic stacking fault energy of 55-70 $\mathrm{mJ} \mathrm{m}^{-2}$ and a twin fault energy of about half this value. ${ }^{26}$ Nonetheless, the high twin density is not unique for PECVD growth, since both, microtwinning and the additional x-ray diffraction peak at $3.007 \mathrm{~nm}^{-1}$ are found in LPCVD grown poly-Si films. ${ }^{20}$ The surprisingly high twin density in PECVD grown $\mu c-\mathrm{Si}: \mathrm{H}$ can be attributed to a particular growth mechanism that will be addressed in a forthcoming publication. Here, we focus on the implications of the specific microstructural features of $\mu c-\mathrm{Si}: \mathrm{H}$ for size and strain measurements from standard $\mathrm{x}$-ray diffraction experiments.

Before assessing the quantitative data from the numerical model for the anisotropic size broadening given above, the simplifying assumptions underlying the model must be discussed in some more detail. Kinematical diffraction by crystals with planar faults has been treated by analytical models for one-dimensional disorder in infinite crystals by several authors, e.g., Refs. 24 and 27-31. A general description by a systematic writing of the Patterson function and its Fourier transform is presented by Cowley ${ }^{29}$ and by Adlhart. ${ }^{31}$ Warren's ${ }^{24}$ Fourier analysis of faulting by stacking disorder or twinning on a single (111) plane is a reference for the line broadening and line shift effects in the case of low fault probability. Our phenomenological approach for the description of the anisotropic diffuse diffraction in $\mu c-\mathrm{Si}: \mathrm{H}$ is not to be considered as yet another treatment of kinematical diffraction in one-dimensional faulted crystals. For the onedimensional problem, it is even less accurate, since it does not include coherent diffraction effects on the intensity of the lattice reflections or streaks. It rather intends to demonstrate the fingerprints of the microstructural disorder in $\mu c-\mathrm{Si}: \mathrm{H}$ 
and related materials. The simplifications (1)-(4) were introduced to get a computable representation of the problem of finite-size effects while accounting for the imperfections of the microcrystalline material, namely, the three-dimensional nature of the faulting, the small size of the coherent domains, the finite size of the single crystal columns and the nonuniformity of these properties in a multitude of grains.

Basically, the assumptions (1)-(4) reflect the perception of finely divided matter, describing the effect of microtwinning as a source of diffuse scattering. Assumptions typically made for finely divided matter are not necessarily valid for nanoparticles with a cluster size of a few hundred atoms. ${ }^{32}$ However, TEM imaging shows that grains in $\mu c$-Si:H with low amorphous volume fraction are considerably larger than the fcc cluster particles described by $\mathrm{Hall}^{32}$ and the symmetry features of fcc bulk particles are fully developed in the electron diffraction patterns and in the x-ray diffractograms, while diffraction signatures peculiar for intertetrahedral structures were not resolved.

The approximation of continuous reciprocal lattice streaks of Gaussian shape appears as another strong simplification regarding the observation of streaks that are composed of single spots in the selected area diffraction patterns of the TEM samples. This approximation is substantiated simply by the considerable number of grains, typically far more than $10^{6}$, that contribute to the $\mathrm{x}$-ray diffractograms: Adlhart's ${ }^{31}$ description of the diffuse kinematical diffraction of lamellar domain structures by domain distribution functions demonstrates that an exponential domain size distribution leads to an exponential decay of the diffraction intensity in the vicinity of the Bragg point in the direction of disorder instead of a distinct satellite pattern. Hence, our simplifications to the problem of computing finite-size effects are well suited for analyzing and retrieving information from the experimental data covering microcrystalline silicon.

By simulating Bragg reflection profiles of two underlying models, lamellar twinned particles and randomly twinned particles, particular finite-size effects to the diffraction from $\mu c-\mathrm{Si}: \mathrm{H}$ have been worked out and are discussed in the following. One of these finite-size effects is an anisotropic broadening with an ordering in the reflection widths of low indexed Bragg peaks, which is characteristic to a particular model in the regime of high twin fault probability $\lambda$. Two types of characteristic ordering are predicted by the numerical simulation and can be seen in the experimental data. In the special case of one-dimensional disorder and low fault probability $\lambda$ our results can be compared with those given by Warre ${ }^{24}$ for twin faulted infinite fcc crystals. Warren ${ }^{24}$ got the following ratios between the apparent grain sizes $\delta_{h k l}$ in powder pattern reflections $\delta_{111}: \delta_{311}: \delta_{220}$ $\approx 1.63: 1.57: 1.00$. This behavior of the size broadening by faulting is very well represented in our simulated data for moderate twinning, i.e., $\delta_{\text {iso }} / \delta_{\text {aniso }}<5$. However, the numerical ratios are somewhat smaller due to the finite particle size $\delta_{\text {iso }}$. For lamellar twinned particles, we observed a change in the ordering to $\delta_{311}>\delta_{220}>\delta_{111}$ in a regime of high twin density, that is not covered by the data given by Warren, ${ }^{24}$ but was found in the experimental data for certain samples.

Typical size data evaluated by our numerical model based on the assumption of finely divided matter is in reasonable agreement with the domain sizes imaged on electron microscopy micrographs. The electron microscopy images generally show coherent twin domains with a size of a few $\mathrm{nm}$, which is usually considerably smaller than the size of a single crystal columnar grain. According to our model, the $\{220\}$ reflection width in $\mathrm{X}$-ray diffractograms can be taken as a reasonable estimate for an upper limit of the global average coherent domain size, disregarding the extreme case of lamellar faulting with high twin density. Taking into account, that the reflection width in x-ray diffraction is not a number weighted measure but a volume weighted measure, ${ }^{33}$ it is conceivable that a typical width $\delta_{220} \approx 5-10 \mathrm{~nm}$ given by the experimentally measured $\mathrm{x}$-ray diffraction patterns is slightly larger than the typical width of the twin lamellae on the TEM images that ranges from a few $\{111\}$ layers to a few $\mathrm{nm}$. Only a small increase of $\delta_{220}$ can be seen upon increase of the crystallinity, i.e., the increase of the column size of a crystalline grain. Indeed, a small increase of $\delta_{220}$ upon increase in the column size, i.e., the increase of $\delta_{\text {iso }}$ is to be expected up to the factor of 2 , since lamellar twin patterns are more pronounced at larger column sizes. Therefore, the twin fault probability $\lambda$ is nearly independent on the growth conditions. The approximate value of $\lambda$ is 0.1 . This result compares nicely to the TEM images of $\mu c-\mathrm{Si}: \mathrm{H}$, which qualitatively reveal a high density of microtwins irrespective of the size of the columnar grains and the crystalline volume fraction. ${ }^{6}$

A twin density $\lambda=0.1$ leads to a significant underestimation of the grain sizes from x-ray diffraction patterns when applying the Scherrer formula without correction by the squeeze factors displayed in Fig. 5. As an example, TEM imaging of the microcrystalline silicon samples covered by the x-ray diffraction patterns in Fig. 3 shows an average width of columnar grains of approximately 50 and $20 \mathrm{~nm}$ at the silane concentration of 2 and $7 \%$, respectively. When taking $\lambda=0.1$, the corresponding values for $\delta_{\text {iso }} / \delta_{\text {aniso }}$ are approximately 15 and 6 . The squeeze factor for the grain size measurement for $\mathrm{x}$-ray diffraction profile widths then ranges between 2.1 and 4.6 for purely lamellar twinned particles and 2.4 and 14.0 for randomly twinned particles, the larger values being related to the larger grain size of $50 \mathrm{~nm}$.

In conclusion, our model calculation yields a reasonable quantitative measure for disorder induced effects in the diffraction patterns of $\mu c-\mathrm{Si}: \mathrm{H}$ due to microtwinning. Inconsistent apparent grain sizes $\delta_{h k l}$ for different $h k l$ in x-ray diffractograms can be attributed to anisotropic broadening of the diffraction linewidths. Twinning on $\{111\}$ lattice planes is a source for disorder induced anisotropic size broadening. Taking the twinning disorder into account, the scatter of apparent grain size data can be explained and can be quantitatively related to complementary TEM data.

The inverse problem however, i.e., the calculation of representative particle sizes and faulting probabilities from experimental diffraction data, is complicated by the combination of the effects of finite sizes, size dispersion and lattice compression or dilation in a single measurement. The numerical calculation of the x-ray reflection profiles clearly demonstrates that a strain analysis from reflections of lattice planes with different orientation is likely to fail for $\mu c-\mathrm{Si}: \mathrm{H}$ 


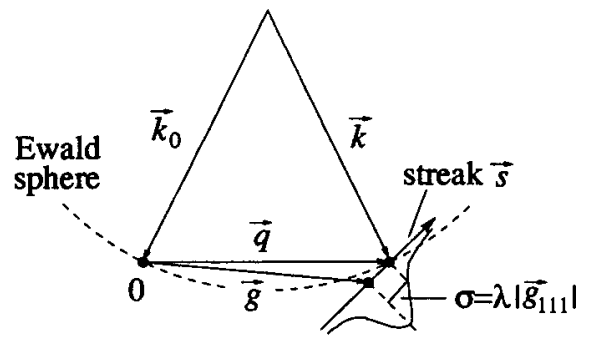

FIG. 6. Ewald construction in reciprocal space illustrating the displacement of Bragg peaks along a streak direction $\vec{s}$. For details see text.

due the presence of a twin domain structure. The character of particle size broadening itself depends not only on the grain size and the twin density. It depends also on the form of the twinning patterns, lamellar or random twinning, which appears inaccessible from x-ray diffraction patterns over a wide range of particle sizes and twin densities. Standard methods of diffraction analyses based on a single particle model are then misleading. Instead, a reasonable model structure must be tested against the experimental data.

\section{CONCLUSIONS}

The most prominent form of disorder in microcrystalline silicon crystalline grains is one- or three-dimensional twinning on $\{111\}$ lattice planes with considerable faulting probability, typically every tenth $\{111\}$ bilayer. A nonmonotoneous order in the apparent grain sizes measured from the widths of different Bragg reflection peaks with increasing magnitude of the scattering vector is characteristic of highly twinned particles. Grain sizes can be estimated from x-ray diffraction patterns correctly only when accounting for anisotropic size broadening due to the microtwins. These finitesize effects must not be confused with interfering strain broadening effects.

\section{ACKNOWLEDGMENTS}

We would like to express our thanks to Dr. P. Hapke and Dr. F. Finger for providing samples and the fruitful discussions on the very high frequency PECVD of microcrystalline silicon.

\section{APPENDIX A: DISPLACEMENT OF BRAGG PEAKS DUE TO STREAKING IN RECIPROCAL SPACE}

Figure 6 illustrates the effect of streaking on the position of Bragg peaks: Bragg reflections will occur for any scattering vector $\vec{q}=\vec{k}-\vec{k}_{0}$ connecting the origin 0 of the reciprocal space and a point on the streak through the reflection $\vec{g}$ of the perfect lattice. $\vec{k}_{0}$ is the wave vector of the incident wave and $\vec{k}$ the wave vector of the diffracted wave. The curve along the streak direction $\vec{s}$ indicates the intensity distribution along the so-called reciprocal lattice rod with a typical width of $\sigma=\lambda\left|\vec{g}_{111}\right|$, i.e., the inverse of the mean width of a twin domain.

The expectation value $\varepsilon$ for the relative displacement of the Bragg peak position $q=|\vec{q}|$ with respect to its position $g=|\vec{g}|$ in the perfect lattice is given by

$$
\varepsilon=\left|1-\frac{q}{g}\right|=\left|1-\frac{\sqrt{g^{2}+2 \sigma \vec{s} \cdot \vec{g}+\sigma^{2}}}{g}\right| .
$$

Neglecting the quadratic term in $\sigma$, expanding the square root for small $\sigma|\vec{s} \cdot \vec{g}| / g^{2} \ll 1$, setting $\vec{s}=1 / \sqrt{3}\langle 111\rangle$ for a twin domain bounded by the (111) lattice plane and expressing $\vec{g}$ in terms of its Miller indices $(h k l)$, one obtains

$$
\varepsilon \approx \frac{\sigma|\vec{s} \cdot \vec{g}|}{g^{2}}=\lambda\left(\frac{h+k+l}{h^{2}+k^{2}+l^{2}}\right) .
$$

\section{APPENDIX B: NUMERICAL CALCULATION OF BRAGG REFLECTION PROFILES IN TWINNED NANOCRYSTALS}

At a twin boundary the atom positions $\vec{R}_{n}$ in Eq. (1) are shifted by a vector $\Delta \vec{r}$. Possible $\Delta \vec{r}$ for a single twin boundary on a $\{111\}$ lattice plane are summarized in Table I. As a consequence, the double sum in Eq. (1) will show an intricate dependence on the individual twin boundary pattern for a single grain and the analytical solution of Eq. (1) is not straight forward. Basically, additional products of phase factors of the form

$$
\exp (2 \pi i \vec{q} \cdot \Delta \vec{r}) \approx \exp (2 \pi i \vec{g} \cdot \Delta \vec{r})
$$

will appear in Eq. (1). As long as the $\vec{g} \cdot \Delta \vec{r}$ do not equal an integer, the phase shifts $2 \pi \vec{g} \cdot \Delta \vec{r}$ will lead to a broadening of the lattice reflection $\vec{g}$ because of loss of phase coherence.

Noninteger $\vec{g} \cdot \Delta \vec{r}$ will occur, e.g., for $\vec{g}=(220)$ when twinning is on the (111) and (111) lattice planes. As a result, the (220) reflection will be affected by twinning on (111) and $(11 \overline{1})$ lattice planes but not by twinning on (1 $\overline{1} 1)$ and (111) lattice planes. In general, $0 \leqslant M \leqslant 4$ different $\{111\}$ twin boundary planes will cause size broadening for a certain Bragg reflection $\vec{g}$.

Given the simplifications (1)-(4) in our phenomenological approach, we avoid the problem of finding an analytical solution for the shape transform $G(\vec{s})$ for a crystallite of a certain orientation, shape and twin boundary pattern within the crystallite. The calculation of the shape transform $|G(\vec{s})|^{2}$ for a single grain is simplified to a convolution of Gaussian profiles

$$
|G(\vec{s})|^{2}=f(\vec{s}) \circledast\left(\sum_{m=1}^{\mathrm{M}} w_{m} h_{m}(\vec{s})\right),
$$

where 


$$
\begin{gathered}
f(\vec{s})=\frac{1}{\sqrt{2 \pi} \sigma_{\text {iso }}} \exp \left(-\frac{|\vec{s}|^{2}}{2 \sigma_{\text {iso }}^{2}}\right), \\
h_{m}(\vec{s})=\frac{1}{\sqrt{2 \pi} \sigma_{\text {aniso }_{m}}} \exp \left(-\frac{\left|\vec{s} \cdot \vec{e}_{m}\right|^{2}}{2 \sigma_{\text {aniso }_{m}}^{2}}\right) .
\end{gathered}
$$

Here, the $w_{m}=1 / M$ are weight factors and $\vec{e}_{m}$ is the twin boundary normal $\left(\left|\vec{e}_{m}\right|=1\right), \sigma_{\text {iso }}$ and $\sigma_{\text {aniso }_{m}}$ are related to the mean grain size $\delta_{\text {iso }}$ and the mean twin domain size $\delta_{\text {aniso }_{m}}$ by the equation $\delta^{-1}=2 \sigma \sqrt{2 \ln 2}$. For the randomly twinned particles, the twin domain size was assumed to be independent on the twin boundary plane, i.e., $\delta_{\text {aniso }_{m}}=\delta_{\text {aniso }}$ for $m=1 \cdots M$.

Equation (B2) gives $|G(\vec{s})|^{2}$ only for a certain orientation of the grain with respect to the diffraction vector $\vec{q}$. However, the excitation vector $\vec{s}$ depends on the diffraction setup, the reciprocal lattice vector and the grain orientation. This becomes clear when expressing $\vec{s}$ as the difference between the scattering vector $\vec{q}$ and the reciprocal lattice vector $\vec{g}$, where $\vec{q}$ depends on the geometry of the diffraction setup and $\vec{g}$ is dependent on the orientation of the grain as shown in Fig. 7.

We can now rewrite the dependence of $|G(\vec{s})|^{2}$ expressing the reciprocal lattice vector $\vec{g}$ in spherical coordinates $\vec{g}$ $=(g, \theta, \varphi):|G(\vec{s})|^{2}=|G[\vec{q},(g, \theta, \varphi)]|^{2}$.

In order to compare the numerical results with experimental data for a nanocrystalline sample, $|G[\vec{q},(g, \theta, \varphi)]|^{2}$ has to be averaged with respect to the orientation distribution function for the grains $p(\theta, \varphi)$ :

$$
\left\langle|G(\vec{q}, g)|^{2}\right\rangle_{p}=\int_{0}^{\pi} \int_{0}^{2 \pi} p(\theta, \varphi)|G[\vec{q},(g, \theta, \varphi)]|^{2} d \theta d \varphi .
$$

For the present calculations, a random number generator (Knuth's ${ }^{34}$ algorithm $P$ ) was used to produce many crystal orientations $(\theta, \varphi)$ to approximate $p(\theta, \varphi)$ and thus the ap-

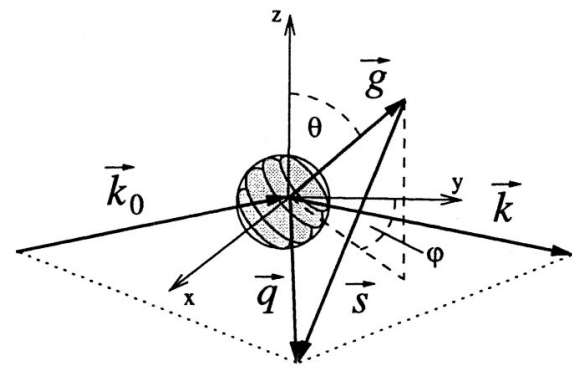

FIG. 7. The relation between the excitation error $\vec{s}$, the scattering vector $\vec{q}$, and the reciprocal lattice vector $\vec{g}=(g, \theta, \varphi) . \vec{k}_{0}$ is the wave vector of the incident wave and $\vec{k}$ the wave vector of the diffracted wave. The relation between the scattering vector $\vec{q}$ and $\vec{k}_{0}, \vec{k}$ is given by $\vec{q}=\vec{k}^{-} \vec{k}_{0}$. A sketch of a spherical crystallite with a stack of lattice planes is projected into the reciprocal space representation.

propriate probability distribution of excitation errors $p\{\vec{s}[\vec{q},(g, \theta, \varphi)]\}$. For each of these orientations, the convolution in Eq. (B2) was calculated by an adaptive integration based on the Simpson rule. The adaptive integration was done with an accuracy better than $1 \%$. Convergence of \langle|$\left.G(\vec{q}, g))\left.\right|^{2}\right\rangle$ was checked during accumulation of crystal orientations, an upper limit of $0.1 \%$ change in \langle|$\left.G(\vec{q}, g))\left.\right|^{2}\right\rangle$ was found typically after averaging about $5 \times 10^{4}$ orientations.

It should be noted, that the orientation of the scattering vector $\vec{q}$ is not identical for different lattice reflections and not parallel to the surface normal in our thin film samples in the case of the asymmetric diffraction set-up, i.e. different multitudes of grains are probed by different reflections. However, this is similar to the symmetric setup, though the diffraction vector $\vec{q}$ is parallel to the foil normal in that case. A divergence in the simulation results for the left side of Eq. (B5) for the symmetric and the asymmetric diffraction setup is not to be expected in the case of a nonrandom orientation of the crystalline grains, i.e., the simulation results presented here are transferable to the case of a symmetric diffraction setup.
${ }^{1}$ K. Yamamoto, M. Yoshimi, Y. Tawada, Y. Okamoto, A. Nakajima, and S. Igari, Appl. Phys. A: Mater. Sci. Process. 69, 179 (1999).

${ }^{2}$ O. Vetterl, F. Finger, R. Carius, P. Hapke, L. Houben, O. Kluth, A. Lambertz, A. Mück, B. Rech, and H. Wagner, Sol. Energy Mater. Sol. Cells 62, 97 (2000).

${ }^{3}$ J. Meier, E. Vallat-Sauvain, S. Dubail, U. Kroll, J. Dubail, S. Golay, L. Feitknecht, P. Torres, S. Fay, D. Fischer, and A. Shah, Sol. Energy Mater. Sol. Cells 66, 73 (2001).

${ }^{4}$ C. C. Tsai, in Amorphous Silicon and Related Materials, edited by H. Fritzsche (World Scientific Publishing, Singapore, 1988), pp. $123-147$.

${ }^{5}$ M. Luysberg, P. Hapke, R. Carius, and F. Finger, Philos. Mag. A 75, 31 (1997).
${ }^{6}$ L. Houben, M. Luysberg, P. Hapke, R. Carius, F. Finger, and H. Wagner, Philos. Mag. A 77, 1447 (1998).

${ }^{7}$ H. Richter, Z.P. Wang, and L. Ley, Solid State Commun. 39, 625 (1981).

${ }^{8}$ R. Tsu, J. Gonzalez-Hernandez, S.S. Chao, S.C. Lee, and K. Tanaka, Appl. Phys. Lett. 40, 534 (1982).

${ }^{9}$ S. Vepřek, F.A. Sarott, and Z. Iqbal, Phys. Rev. B 36, 3344 (1987).

${ }^{10}$ A. Matsuda, J. Non-Cryst. Solids 59\&60, 767 (1983).

${ }^{11} \mathrm{~S}$. Veprek, in Materials Issues in Microcrystalline Semiconductors, Vol. 164 of MRS Symposia Proceedings, edited by P. M. Fauchet, K. Tanaka, and C. C. Tsai (Materials Research Society, Pittsburgh, 1990), p. 39. 
${ }^{12}$ N. Shibita, K. Fukuda, H. Ohtoshi, J. Hanna, S. Oda, and I. Shimizu, in Amorphous Silicon Technology-1987, Vol. 95 of MRS Symposia Proceedings (Materials Research Society, Pittsburgh, 1987), p. 225.

${ }^{13}$ F. Finger, R. Carius, P. Hapke, L. Houben, M. Luysberg, and M. Tzolov, in Amorphous Silicon Technology-1997, Vol. 452 of MRS Symposia Proceedings (Materials Research Society, Pittsburgh, 1997), p. 725.

${ }^{14}$ P. Hapke and F. Finger, J. Non-Cryst. Solids 227-230, 861 (1998)

${ }^{15}$ M. Hart and W. Parrish, Mater. Sci. Forum 9, 39 (1986).

${ }^{16}$ T.C. Huang, Adv. X-Ray Anal. 33, 91 (1990).

${ }^{17}$ C.B. Carter, Philos. Mag. A 50, 133 (1984).

${ }^{18}$ Y. Lereah and E. Grünbaum, Philos. Mag. A 50, 1 (1984).

${ }^{19}$ M. Hendriks, S. Radelaar, A.M. Beers, and J. Bloem, Thin Solid Films 113, 59 (1984).

${ }^{20}$ H. Cerva, J. Mater. Res. 6, 2324 (1991).

${ }^{21}$ R. Kobliska and S. Solin, Phys. Rev. B 8, 3799 (1973).

${ }^{22}$ L. Houben, M. Luysberg, R. Carius, P. Hapke, and H. Wagner, in Microscopy of Semiconducting Materials, edited by A. G. Cullis and R. Beanland (IOP, Bristol, 1999), vol. 164, p. 211.

${ }^{23}$ J.I. Langford and D. Louër, Rep. Prog. Phys. 59, 131 (1996).

${ }^{24}$ B. E. Warren, X-ray Diffraction (Addison-Wesley, Reading, MA, 1969).

${ }^{25}$ M.H. Hong, A.V. Samant, and P. Pirouz, Philos. Mag. A 80, 919 (2000).

${ }^{26}$ A. Gross and H. Teichler, Philos. Mag. B 64, 413 (1991).

${ }^{27}$ H. Jagodzinski, Acta Crystallogr. 2, 201 (1949).

${ }^{28}$ H. Jagodzinski, Acta Crystallogr. 2, 208 (1949).

${ }^{29}$ J.M. Cowley, Acta Crystallogr., Sect. A: Cryst. Phys., Diffr., Theor. Gen. Crystallogr. 32, 83 (1976).

${ }^{30}$ J.M. Cowley, Acta Crystallogr., Sect. A: Cryst. Phys., Diffr., Theor. Gen. Crystallogr. 32, 88 (1976).

${ }^{31}$ W. Adlhart, Acta Crystallogr., Sect. A: Cryst. Phys., Diffr., Theor. Gen. Crystallogr. 37, 794 (1981).

${ }^{32}$ B.D. Hall, J. Appl. Phys. 87, 1666 (2000).

${ }^{33}$ C.E. Krill and R. Birringer, Philos. Mag. A 77, 621 (1998).

${ }^{34}$ D. E. Knuth, Art of Computer Programming (Addison Wesley, Reading, MA, 1997), Vol. 2. 